\title{
A deformable plate interacting with a non-Newtonian fluid in three dimensions
}

\author{
Luoding $\mathrm{Zhu}^{\diamond}$, Xijun $\mathrm{Yu}^{\star}$, Nansheng $\mathrm{Liu}^{\Delta}$, Yongguang $\mathrm{Cheng}^{\dagger}$, Xiyun $\mathrm{Lu}^{\Delta}$, \\ $\diamond$ Department of Mathematical Sciences, Indiana University-Purdue University Indianapolis, Indianapolis, \\ IN 46202, USA. corresponding author, luozhu@iupui.edu, (317)278-9225(O), (317)274-3460(Fax). \\ * Laboratory of Computational Physics, Institute of Applied Physics \\ and Computational Mathematics, Beijing, PR China 10088. \\ $\Delta$ Department of Modern Mechanics, University of Science and Technology of China, Hefei, Anhui 230026, \\ China \\ $\dagger$ State Key Laboratory of Water Resources and Hydropower Engineering Science, Wuhan University, \\ Wuhan, PR China 430072.
}

\begin{abstract}
We consider a deformable plate interacting with a non-Newtonian fluid flow in three dimensions as a simple model problem for fluid-structure-interaction (FSI) phenomena in life sciences (e.g., red blood cell interacting with blood flow). A power-law function is used for the constitutive equation of the non-Newtonian fluid. The lattice Boltzmann equation (the D3Q19 model) is used for modeling the fluid flow. The immersed boundary (IB) method is used for modeling the flexible plate and handling the fluid-plate interaction. The plate drag and its scaling are studied; the influences of three dimensionless parameters (power-law exponent, bending modulus, and generalized Reynolds number) are investigated.
\end{abstract}

Key words: immersed boundary method, lattice Boltzmann model, fluid-structure-interaction, non-Newtonian fluid, deformable solid, drag reduction and scaling

\section{Introduction}

Problems involving interactions between a deformable solid and an incompressible fluid are very rich in biology: swimming of fish [44], flying of birds [37], flowing of erythrocytes in arteries and veins [48], and cytoskeleton moving in cytoplasm inside a cell [33], to name but a few. The fluid may be Newtonian (e.g., air or water) or non-Newtonian (e.g., blood or cytoplasm). The solid moves with the flow because of fluid viscosity; yet the solid experiences drag because of the force exerted on the surface of solid by the fluid.

Classic theory in fluid mechanics [5] has showed that the drag coefficient for a rigid solid scales as the reciprocal of the flow Reynolds number $(R e)$ for small values of $R e$ and the total drag scales as the second power of the incoming flow-speed for sufficiently large $R e$. Alben et al. $[1,2]$ have discovered that the drag of a one-dimensional flexible fiber immersed in a two-dimensional inviscid flow is proportional to the $\frac{4}{3}$ power of the incoming flow-speed. Zhu [52] has found that the scaling is indeed Reynolds-number dependent: the exponent goes monotonically from nearly 2 towards $4 / 3$ as $R e$ goes from 10 to 800 . Zhu et al. [53] has

Preprint submitted to Physics of Fluids

July 18, 2017

This is the preprint of the article published in final edited form as:

Zhu, L., Yu, X., Liu, N., Cheng, Y., \& Lu, X. (2017). A deformable plate interacting with a non-Newtonian fluid in three dimensions. Physics of Fluids, 29(8), 083101. https://doi.org/10.1063/1.4996040 
found that the drag of $2 \mathrm{D}$ deformable sheet is approximately proportional to the incoming flow-speed for Reynolds numbers in $[1,100]$ in three dimensions.

However, to the best of our knowledge, the drag of a deformable body in a non-Newtonian fluid flow is not studied. Because non-Newtonian fluids are qualitatively different from Newtonian fluids in several aspects, it appears not reasonable to assume that the existing results of drag and its scaling are applicable to non-Newtonian fluids. For instance, due to the presence of elasticity (hence elastic force) in non-Newtonian fluid, the drag of a body in non-Newtonian fluid flow should be different from a very viscous fluid where the elasticity (hence the elastic component of drag) is absent. Drag and drag scaling involving nonNewtonian fluid can be important to many biological problems involving FSI. For instance, red blood cells in a severely stenosed artery may get damaged because of the excessively elevated force from the flow. Therefore, in this work, we study fluid-structure-interaction involving non-Newtonian fluids.

Because of the high level of complexity of FSI problems, analytical solutions are very rare; instead, numerical solutions by a computational approach are obtainable. There are various computational methods in the literature specifically designed for FSI problems. The first such a method is probably the immersed boundary (IB) method pioneered by Charles S. Peskin [35, 36] in 1970s'. Other methods include the immersed interface method [26, 27, 28] and the immersed finite element method [54, 29]. Note that there exists another class of IB methods that are specifically designed for fluid-rigid-body-interactions where the motion of structure is given in advance $[43,46,32,4]$. See more relevant numerical methods cited in $[53]$.

There exist various versions of the IB method, too, including the formally $2^{\text {nd }}$-order versions $[25,15]$, the penalty version [22], and the non-Newtonian version [10, 11]. See more versions of the IB methods cited in [53]. In this work, we extend the lattice-Boltzmann based immersed boundary (LB-IB) method developed by Zhu et al. [53] to the case of nonNewtonian fluids. The major differences between our method and the existing method in $[10,11]$ are as follows: the existing method is two dimensional and is formulated for Oldroyd-B fluid; the Navier-Stokes equations are solved by a fractional step project method of Kim and Moin [23] and the Oldroyd-B model is solved by the Stokes Oldroyd-B scheme presented by [41]. While in our work, the method is three dimensional and is formulated for a power-law fluid; the Navier-Stokes equations together with the power-law constitutive equation are solved by the lattice Boltzmann D3Q19 model. A similar method has been developed by Tian [42] in two dimensions.

Fluids can be broadly classified as Newtonian fluids and non-Newtonian fluids. NonNewtonian fluids have many distinct properties, such as shear-thinning and normal stress differences. Modeling non-Newtonian fluids is still quite challenging, partly because of the different constitutive equations describing the stress-strain relationships and history effects. To date, there exists no uniform formulation for all non-Newtonian fluids. In this work, we start from non-Newtonian fluids whose constitutive equations can be modelled by power law functions. Such fluids include the blood and cytoplasm.

The lattice Boltzmann (LB) method is an alternative approach for solving Navier-Stokes equations. It has been a popular method in Computational Fluid Dynamics (CFD) [38, 6, $20,17,18,30,19,45,31,39]$. The advantages of the LB method include relative simplicity in parallelization, modeling extra flow physics, and handling highly complex rigid flow boundary 
such as porous media. The first IB method using the LB approach was developed by Feng and Michaelides [12, 13]. Other similar LB-IB methods include [40, 14, 47, 34, 24, 9]. In this work, we extend the LB-IB method introduced by Zhu et al. [53] for modeling and simulation of non-Newtonian-fluid deformable-structure interactions. Due to the complexity of FSI problems, we start from an ideal model problem to investigate the drag and its scaling of a deformable structure in a non-Newtonian fluid. The model problem involves a two-dimensional flexible plate tethered at its midline interacting with a viscous flow of non-Newtonian fluid whose constitutive equation is given by a power function.

There exist several dimensionless parameters in the model problem. The bending rigidity of the plate $\hat{K}_{b}$, the exponent $n$ of the power-law (fluid constitutive equation), the mass ratio of the plate and fluid $M$, and a dimensionless ratio similar to the Reynolds number of Newtonian flow. For simplicity, this ratio is called generalized Reynolds number and denoted by $R e g$ in this paper (see next section for definition). In many FSI problems in life sciences, the structure is neutrally buoyant in the ambient fluid, therefore the value of $M$ is fixed to 1 in this study. All other parameters are variable. Series of simulations are performed with one dimensionless parameter varied and others fixed. The drag of the plate is computed and its position is recorded. The drag coefficients with respect to $n, R e, \hat{K}_{b}$ are studied for several fixed values of other parameters. The drag scaling with respect to inflow speed is investigated for different values of $n$ and $R e$.

The remaining of the paper is structured as follows. Section 2 outlines the physical model problem. Section 3 gives the mathematical formulation of the model problem. Section 4 briefly address the extension of the LB-IB method to the non-Newtonian-fluid structure interaction. Section 5 presents the major numerical results. Section 6 concludes the article by a summary and discussion.

\section{The model problem}

We consider a 3D viscous flow of non-Newtonian fluid past a flexible plate tethered at the midline in a rectangular channel. A right-handed Cartesian coordinate system is used with the $\mathrm{x}$-axis pointing from left-to-right (i.e. main flow direction) and the y-axis pointing from front to rear. A quadrilateral deformable plate is introduced near the left boundary at the center of the channel cross-section. The plate is tethered at its midline and otherwise unconstrained. No-slip boundary condition is imposed on the front, rear, top, and bottom. Inflow and outflow boundary conditions are imposed on the left and right, respectively (uniform flow with speed $V_{0}$ ). The fluid is assumed to be viscous, homogeneous, incompressible, and non-Newtonian. The rectangular flexible plate is made of two orthogonal families of linearly elastic fibers (along longitudinal and lateral directions) that possess identical mechanical properties.

The flow channel has a square cross-section and has a length-width ratio of 2. The ratio of width to length of the elastic plate is 1 to 2 . Stiff springs are used to tether the plate midline to a fixed line-segment joining two points $\left(x_{t}, y_{1 t}, z_{t}\right)$ and $\left(x_{t}, y_{2 t}, z_{t}\right)$. The plate width is $W=y_{2 t}-y_{1 t}$. The plate length is $L=2 W$. The plate is initially placed vertically, i.e., on the y-z plane. See Fig. 1 for illustration of the model problem.

Table 1 lists three major dimensionless flow parameters of the problem and their values

used in the simulations. Here $V_{0}$ is the inflow speed with unit $m / s, \rho_{0}$ is the fluid mass 
density with unit $\mathrm{kg} / \mathrm{m}^{3}, m_{p}$ is a constant of the power-law model with unit $P a \cdot s^{n}, K_{b}$ is the plate fiber bending rigidity with unit $N \cdot m^{2}, \mu(\dot{\gamma})$ is the fluid dynamical viscosity with unit $\mathrm{kg} /(\mathrm{m} \cdot \mathrm{s})$, and $\dot{\gamma}$ is the flow shear rate with unit $\mathrm{s}^{-1}$ (see next section for its definition). The dimensionless variable $n$ is the exponent of the power-law of the non-Newtonian fluid. Other dimensionless parameters used in the work are as below: the fiber compression/stretching coefficient $\hat{K}_{s}=20$; that of the tethering virtual springs $\hat{K}_{s t}=40$.

Notice that 1) The unit of parameter $m_{p}$ is $P a \cdot s^{n}$. Therefore the quantity $m_{p}$ may not have any physical meanings for power-law fluid. To make a distinction between Newtonian and non-Newtonian fluids, we shall make explicit use of the name generalized Reynold number, Reg, for the dimensionless group associated with non-Newtonian fluid. It should be pointed out that this dimensionless group has incorporated non-Newtonian effect of the power-law fluid. 2) The plate is made of identical fibers that are homogeneous and possess the same mechanical properties. For simplicity the dimensionless group $\hat{K}_{b}$ is defined using the bending rigidity of the constitutive fiber.

\begin{tabular}{|c|c|c|}
\hline Name & Definition & Range \\
\hline Generalized Reynolds Number $(\operatorname{Reg})$ & $\frac{\rho_{0} V_{0}^{2-n} W^{n}}{m_{p}}$ & $0.01-200$ \\
\hline Flexure Modulus $\left(\hat{K}_{b}\right)$ & $\frac{K_{b}}{\rho_{0} V_{0}^{2} W^{4}}$ & $0.0001-0.008$ \\
\hline Power law exponent $(n)$ & $\mu(\dot{\gamma})=m_{p}(\dot{\gamma})^{n-1}$ & $0.5-1.9$ \\
\hline
\end{tabular}

Table 1: Parameter ranges (dimensionless) used in simulation.

\section{The mathematical formulation of the model problem}

The dimensionless mathematical formulation of the above model problem is as follows:

$$
\frac{\partial g(\mathbf{x}, \boldsymbol{\xi}, t)}{\partial t}+\boldsymbol{\xi} \cdot \frac{\partial g(\mathbf{x}, \boldsymbol{\xi}, t)}{\partial \mathbf{x}}+\mathbf{f}(\mathbf{x}, t) \cdot \frac{\partial g(\mathbf{x}, \boldsymbol{\xi}, t)}{\partial \boldsymbol{\xi}}=-\frac{1}{\tau}\left(g(\mathbf{x}, \boldsymbol{\xi}, t)-g^{(0)}(\mathbf{x}, \boldsymbol{\xi}, t)\right)
$$

The motions of the plate and fluid are described by a simplified Boltzmann equation (Eq. 1), i.e., the Bhatnagar-Gross-Krook (BGK) equation [3]. The function $g(\mathbf{x}, \boldsymbol{\xi}, t)$ represents the velocity distribution function of a single particle at space $\mathbf{x}$, in time $t$, and with particle velocity $\boldsymbol{\xi}$. The complex collision operator in the Boltzmann equation is approximated by the term $-\frac{1}{\tau}\left(g-g^{(0)}\right)$, where the $\tau$ is the relaxation time. It is connected to the fluid kinematic viscosity $\nu$ in the LB method. This is the well-known BGK approximation [3]. For the D3Q19 model we use, $\nu=\frac{2 \tau-1}{6}$. The $g^{(0)}$ is the Maxwellian distribution. The external force term $\mathbf{f}(\mathbf{x}, t)=\mathbf{f}_{i b}(\mathbf{x}, t)+\mathbf{f}_{e x t}(\mathbf{x}, t)$. The $\mathbf{f}_{i b}(\mathbf{x}, t)$ is the force exerted by the plate to the fluid. The $\mathbf{f}_{e x t}(\mathbf{x}, t)$ is other external forces applied on the fluid such as gravity. As in the statistical mechanics, the velocity distribution function $g$ can be used to compute the macroscopic variables: fluid mass density $(\rho)$ and momentum $(\rho \mathbf{u})$. Note that in Eq. 1 the kinematic viscosity $\nu$ is constant (i.e. Newtonian fluid).

The constitutive law of non-Newtonian fluids considered in our work may be written as a power-law function: $\mu(\dot{\gamma})=m(\dot{\gamma})^{n-1}$, where $\mu$ is the fluid dynamical viscosity, $\dot{\gamma}$ is 
the flow shear rate which is defined as $\dot{\gamma}=\sqrt{2 D_{i j} D_{i j}}$, where $D_{i j}=\frac{1}{2}\left(u_{i j}+u_{j i}\right)$, here $u_{i j}$ means $\frac{\partial u_{i}}{\partial x_{j}}\left(u_{i}\right.$ is the $i^{t h}$ component of the flow velocity). Constants $m$ and $n$ are model parameters describing the properties of the non-Newtonian fluid: $n<1$ characterizes shearthinning or pseudoplastic fluids such as blood, cytoplasm, and paint; $n>1$ characterizes shear-thickening or dilatant fluids such as oobleck (a mixture of cornstarch and water); and $n=1$ characterizes Newtonian fluids.

To introduce the power-law model into the LB-IB framework [53], we take advantage of the new dimensionless parameter $(R e g)$ which corresponds to the Reynolds number for Newtonian fluids, as defined in the previous section. Given the value of $R e g$ and $n$, the value of $m_{p}$ in lattice Boltzmann units (to be used in LB simulation) may be computed from the definition of Reg, as listed in Table 1.

The shear rate is computed in lattice Boltzmann D3Q19 model as follows: $\dot{\gamma}=\sqrt{2 S_{\alpha \beta} S_{\beta \alpha}}$. Here $S_{\alpha \beta}(\alpha=1,2,3 ; \beta=1,2,3)$ is computed by $S_{\alpha \beta}=-\frac{3}{2 \tau} \sum_{i=0}^{i=18} \xi_{i \alpha} \xi_{j \beta} f_{i}^{(1)}$, where $f_{i}^{(1)}=$ $f_{i}-f_{i}^{(0)}$. Here $f_{i}$ is the single particle velocity distribution function and $f_{i}^{(0)}$ is the equilibrium distribution function, and $\xi_{i \alpha}(i=0,1, \ldots, 18 ; \alpha=1,2,3)$ is the discrete direction of the D3Q19 model along which particles are allowed to move or to stay. Note that because fluid viscosity is shear rate dependent for the non-Newtonian fluid, the relaxation time $\tau$ is no longer a constant in the LB model; instead it is a variable varying in both space and time.

Eq. 2 is used to compute the Eulerian force density $\mathbf{f}_{i b}(\mathbf{x}, t)$, which is defined on the fixed Eulerian lattice, from the Lagrangian force density $\mathbf{F}(\boldsymbol{\alpha}, t)$, which is defined on the Lagrangian grid. This is done for any constitutive fiber point of the immersed plate whose Lagrangian coordinate is $\boldsymbol{\alpha}$ :

$$
\mathbf{f}_{i b}(\mathbf{x}, t)=\int \mathbf{F}(\boldsymbol{\alpha}, t) \delta(\mathbf{x}-\mathbf{X}(\boldsymbol{\alpha}, t)) d \alpha
$$

Where the function $\delta(\mathbf{x})$ is the Dirac $\delta$-function; the $\mathbf{F}$ is Lagrangian force density which can be computed as follows:

$$
\mathbf{F}(\boldsymbol{\alpha}, t)=-\frac{\partial \mathcal{E}}{\partial \mathbf{X}}=-\frac{\partial\left(\mathcal{E}_{s}+\mathcal{E}_{b}\right)}{\partial \mathbf{X}}
$$

In Eq. 3, $(\mathcal{E})$ is the elastic potential energy which has two contributions: compression/stretching contribution $\left(\mathcal{E}_{s}\right)$ and a bending contribution $\left(\mathcal{E}_{b}\right)$. For a typical constitutive fiber, these energies are defined respectively by Eq. 4 and Eq. 5:

$$
\begin{gathered}
\mathcal{E}_{s}=\frac{1}{2} K_{s} \int\left(\left|\frac{\partial \mathbf{X}}{\partial \beta}\right|-1\right)^{2} d \beta \\
\mathcal{E}_{b}=\frac{1}{2} K_{b} \int\left|\frac{\partial^{2} \mathbf{X}(\beta, t)}{\partial \beta^{2}}\right|^{2} d \beta
\end{gathered}
$$

The symbol $\beta$ is the Lagrangian coordinate of the fiber. The coefficients of stretching/compression $\left(K_{s}\right)$ and bending $\left(K_{b}\right)$ are both constant and associated with the plate Young's modulus. 
A first-order system of ordinary differential equations (Eq. 6) is used to update the position of the immersed plate:

$$
\frac{\partial \mathbf{X}}{\partial t}(\boldsymbol{\alpha}, t)=\mathbf{U}(\boldsymbol{\alpha}, t)
$$

The $\mathbf{X}(\boldsymbol{\alpha}, t)$ is the position of the plate (i.e. Eulerian coordinate) with Lagrangian coordinate $\boldsymbol{\alpha}$ and at time $t$. Using the same $\delta$-function (as in Eq. 2 for transferring the plate force to the fluid), the velocity of the immersed boundary $\mathbf{U}(\boldsymbol{\alpha}, t)$ is interpolated from that of the fluid $\mathbf{u}(\mathbf{x}, t)$ :

$$
\mathbf{U}(\boldsymbol{\alpha}, t)=\int \mathbf{u}(\mathbf{x}, t) \delta(\mathbf{x}-\mathbf{X}(\boldsymbol{\alpha}, t)) d \mathbf{x}
$$

\section{Numerical Method}

The mathematical formulation of the problem (Eqs. 1 - 7) is discretized on a uniform fixed Eulerian grid for the fluid with uniform spacing h (the number of grid nodes is $N_{x}, N_{y}$ and $N_{z}$ in x, y and z direction, respectively), plus a set of moving Lagrangian points for the plate with meshwidth $\Delta \alpha_{1}=\Delta \alpha_{2}=\frac{1}{2} h$. The BGK equation Eq. 1 is discretized by the LB D3Q19 model [38, 6], as follows:

$$
g_{j}\left(\mathbf{x}+\boldsymbol{\xi}_{j}, t+1\right)=g_{j}(\mathbf{x}, t)-\frac{1}{\tau}\left(g_{j}(\mathbf{x}, t)-g_{j}^{0}(\mathbf{x}, t)\right)+\left(1-\frac{1}{2 \tau}\right) w_{j}\left(\frac{\boldsymbol{\xi}_{j}-\mathbf{u}}{c_{s}{ }^{2}}+\frac{\boldsymbol{\xi}_{j} \cdot \mathbf{u}}{c_{s}{ }^{4}} \boldsymbol{\xi}_{j}\right) \cdot \mathbf{f}
$$

In the D3Q19 model, particles at each lattice node can move (impinging and exiting) along 18 different directions. They may also stay at the node. Therefore, the velocity space of particles is discretized by 19 different directions: $\boldsymbol{\xi}_{j}, j=0,1, \ldots, 18$. Along each such direction at a node, impinging or exiting, $g_{j}, g_{j}^{0}, w_{j}$ are particle velocity distribution function, equilibrium distribution function, and weight of the LB model, respectively. The external forces of Eq. 8 is treated by the approach of Guo et al. [16]. Here the fluid velocity $\mathbf{u}$, forces f are evaluated at time $t$.

The no-slip boundary condition for fixed rigid walls are modeled by the bounce-back scheme [7]. Notice that the freely-moving immersed flexible plate does not need any special treatment by the LB method. It is instead treated by the IB method: the fluid "feels" the existence of the flexible plate through the force.

The verification and validation of the numerical method have been essentially performed in the previous works $[49,53]$. The new feature introduced in the method is the shear-ratedependent fluid kinematical viscosity, which is incorporated algebraically into the method and code.

\section{Major computational results}

\subsection{Visualization of flow and plate}

Visualizations on the flow and plate are performed by plotting streamlines. A typical picture is shown in Figs. 2. The dimensionless parameters are as follows: $R e=120, \hat{K}_{b}=$ $0.005, n=0.6$. The dimensionless time is 50,000 in lattice Boltzmann unit. The flexible 
plate at a quasi-stationary state is visualized by the gray surface. A streamline is visualized by a thick colorful curve. Its tangent represents velocity direction and its color represents velocity magnitude. One can see complicated patterns of the curves in the region close to the flexible plate (compared to other regions), particularly right in front of and behind the plate: some lines are winding and twining. This indicates formation of vortices and demonstrates the flow complexity near the plate. Note that the seemingly intersecting lines in the 3D plot in fact do not intersect.

We also try to visualize the vortical field by iso-surfaces of vorticity and the Q-value. However, the results are not as satisfactory as in our previous work [53]: vorticity concentrates primarily on the plate and vortex shedding is not obvious. Possible reasons include: 1) the generalized Reynolds number is not high enough; 2) the fluid elasticity of non-Newtonian fluid may prevent the vorticity formation and vortex shedding.

\subsection{Coefficient and scaling of plate drag}

The instantaneous drag of the plate is computed by adding up the tension forces of the virtual springs connecting all the discrete points of the plate middle line to the fixed spatial points. Note that this simple approach in the IB methods is equivalent to integration of viscoelastic fluid force on the entire plate surface. The virtual spring is given a very large value of stiffness such that the plate midline is virtually immobile in space (the maximum magnitude of the displacement of the midline nodes is less than $10^{-2}$ in LB unit for length).

The drag coefficient averaged in time is computed by

$$
\bar{C}_{d}=\frac{\bar{D}}{0.5 \rho_{0} V_{0}^{2} L W}
$$

Where $\bar{D}$ is the plate drag averaged in time; $L$ and $W$ are the initial plate length and width, respectively. The instantaneous drag $D$ is computed and stored every $t_{r}$ steps (in LB unit) from the beginning $(t=0)$ to the end of a simulation $\left(t_{e}\right)$. The average of $D$ is performed starting at an instant $t_{s}$ when the flow-plate system reaches a quasi-steady state until $t_{e}$. In our simulations, $t_{s}=100,000, t_{e}=200,000$, and $t_{r}=200$. Three sets of simulations were designed and run with respect to three most important dimensionless parameters: the exponent $n$ of the non-Newtonian fluid, the generalized Reynold number Reg of the flow, and the bending modulus of the elastic plate $\hat{K}_{b}$. See Table 1 for the ranges of these parameters used in the work. For the series with varying values of $n$, three different values of Reg $(1,10,100)$ are used, and the bending modulus is fixed to $\hat{K}_{b}=0.001$. For the series with varying values of $R e g$, five different values of $n=0.5,0.75,1.0,1.25,1.75$ are used, and the bending modulus is fixed to $\hat{K}_{b}=0.001$. For the series with varying values of $\hat{K}_{b}$, four different values of $n=0.6,0.9,1.25,1.5$ and three different values of generalized Reynolds number $(\operatorname{Reg}=1,10,100)$ are used.

\subsubsection{Drag coefficient $\bar{C}_{d}$ versus power law exponent $n$}

Three groups of data of drag coefficient $\left(\bar{C}_{d}\right)$ versus exponent $n$ for three distinct generalized Reynolds numbers are plotted in the top panel of Fig. 3. The blue square, red triangle, and black dot represent drag coefficient data for $R e=1,10,100$, respectively. From Fig. 3, we see that, within the parameter ranges used in the work, the drag coefficient increases with 
the increase of $n$ for a fixed value of $R e$ and it decreases with the increase of $R e$ for a fixed value of $n$. Drag coefficient decreases significantly with the increase of $R e$ at the beginning; and later decreases slightly when $R e$ is sufficiently large (greater than 10).

Plotted on the bottom panels of Fig 3 are the plate position and shape when it settles down into a quasi-stationary state for different values of $n$ with $R e=1,10,100$, from left to right, respectively. For any panel in the bottom (corresponding to a specific value of $R e$ ), a plate with a different value of $n$ is identified by a surface of different color (the surface may look like a line at certain reviewing angles). One can notice that some plates look as if they were overlapped. This is because their positions and shapes are very close to each other but they are not actually identical. The correspondence between the value of $n$ and the color is not given here. This information is not informative because it is difficult to show such a one-to-one connection in the figure. The reasons may include 1) there are only seven different colors available in Matlab so a color has been used more than once for different values of $n ; 2)$ the shape and position of plate with some values of $n$ are not always well separated physically: they have more complex shapes than just a piece of rigid plate bent along the middle line; some may partially overlap and cross each other; 3)these are 3D plots; not all features/aspects can be displayed from a given angle. Nevertheless, these figures are still used for a different purpose - providing a qualitative profile/envelope of the collection of multiple plates with different generalized Reynolds numbers. Similar explanation applies to Fig. 4 and Fig. 5 but shall not be repeated.

By comparison of the three panels on the bottom of Fig. 3, one can see that as Re increases, the profile of plates with different $n$ generates a greater envelope (representing a greater obstacle in the flow). Therefore the shape drag of the plate becomes greater as $R e$ increases. Yet the data on the top panel shows that the drag coefficient becomes smaller as $R e$ increases. This indicates that the total drag is dominated by viscoelastic drag, rather than shape drag.

\subsubsection{Drag coefficient $\bar{C}_{d}$ versus generalized Reynolds number Reg}

The top panel in Fig. 4 plots five sets of drag coefficient $\left(\bar{C}_{d}\right)$ data (time-averaged) versus $R e g$ on a log-log scale for five different values of the power law exponent $n$. Symbols (dot, square, plus, triangle, and star) with different colors represent drag coefficient data for different value of $n$ (see figure legend.) From Fig. 4, we see that, within the parameter ranges used, the drag coefficient decreases with the increase of $R e$ for a fixed value of $n$ and it increases with the increase of $n$ for a fixed value of $R e g$.

Plotted on the bottom panels of Fig 4 are plate position and shape when it attains a quasi-stationary state for different values of $\operatorname{Reg}$ with $n=0.5,0.75,1.0,1.25,1.75$, from left to right, respectively. For any panel in the bottom (corresponding to a specific value of $n$ ), each surface with a different color visualizes a plate with a different Reg (some surface may look like a line when seen at certain angles). One can notice the same overlapping phenomenon as in Fig. 3 and the explanation is the same. The surface located the inner most is associated with the smallest Reg and the surface located the outer most is associated with the greatest Reg. With the increase of Reg, the plate bends less and its final position moves outwards. Then it appears that the drag coefficient should become greater which is not the case from our simulations. Again this indicates that the bending of the plate is dominated by the fluid viscoelastic forces rather than the flow inertial forces. Smaller generalized Reynolds number 
leads to greater viscoelastic force; thus the drag coefficient is greater (although the shape drag is less).

Comparison of the five panels on the bottom of Fig. 4 shows that as $n$ increases, the collection of the plates with different $R e$ forms a slightly smaller envelop (representing a slightly smaller obstacle in the flow). This is because the drag increases with $n$.

Figs 3 and 4 show that, compared to the Newtonian case $(n=1)$, the drag of the plate is increased for $n \geq 1$, but decreased for $n \leq 1$. In another word, the shear thinning fluid induces drag reduction, but the shear thickening fluid induces drag increase. In both cases, the drag coefficient increases monotonically with the value of $n$.

\subsubsection{Drag scaling}

Dimensionless drag related variable $D$ and bending-modulus related variable $\eta$ are introduced to gauge the drag and flexibility of the plate, as in $[1,2]$. They are defined as:

$D=\bar{C}_{d} \eta^{2}$ and $\eta=\frac{1}{\sqrt{\hat{K}_{b}}}$. Notice that this is not the physical dimensionless drag of the plate because of the factor $\eta^{2}$ involved. Instead, introduction of the variable is for obtaining the drag scaling between the dimensional total drag with respect to the inflow speed $V_{0}$. Also notice that a more flexible plate has a greater value of $\eta$ but a smaller value of $\hat{K}_{b}$. As indicated in $[1,2]$, the dimensional total plate drag scales with the inflow speed $V_{0}$ the same way as $\mathrm{D}$ scales with $\eta$, i.e., the exponent of the power law is the same. Therefore, the scaling laws in Fig 5 between $D$ and $\eta$ are the same as those between the total drag and inflow speed.

The top panels of Fig 5 plots the dimensionless drag $D$ versus plate flexibility $\eta$ with four different values of $n$ on a log-log scale for three different generalized Reynolds numbers $R e=1,10,100$. The red dot, blue circle, cyan triangle, and black star represent respectively results for $n=0.6,0.9,1.25,1.5$. The four straight lines with colors red, blue, cyan, and black are the fitted lines to the data by the method of least square for any given value of $n$. Slopes of these lines are approximated as $1.9(\operatorname{Reg}=1), 1.6(\operatorname{Reg}=10)$, and $1.2(\operatorname{Reg}=100)$. This suggests that the drag function $\mathrm{D}(\eta)$ can be approximated by a power function for a given value of $n$, and the exponents are approximately 1.9 for $R e g=1,1.6$ for $R e g=10$, and 1.2 for $R e g=100$. The exponent of drag scaling seems to be insensitive to $n$.

In contrast to the results on drag scaling of rigid and deformable body in Newtonian fluids (see Introduction), our simulations appear to indicate that the drag of a deformable plate in a 3D non-Newtonian flow scales approximately as the second power of the inflow speed when Reg is sufficiently small (around 1); and approximately as the first power of inflow speed when $R e g$ is sufficiently large (around 100). The power decreases from 2 to 1 as Reg increases from approximately 1 to 100. Our results indicate the drag scaling is insensitive to the power-law exponent $n$ of the non-Newtonian fluid.

The bottom panels of Fig. 5 give the plate position and shape after it settles down into a quasi-stationary state for different values of bending modulus $\hat{K}_{b}$ with $n=0.6,0.9,1.25,1.5$ for $R e=10$ (profiles are similar for other generalized Reynolds numbers). Each surface of a color is associated with a plate with a different value of $\hat{K}_{b}$. Some surfaces may look like lines when seen at certain angles. The surface situated inner most is associated with the smallest $\hat{K}_{b}$ and the surface situated outer most is associated with the largest $\hat{K}_{b}$. As $\hat{K}_{b}$ increases the plate bends less and its final position expands outwards. This is because a 
plate with smaller value in $\hat{K}_{b}$ is more flexible and gets bent and streamlined by the local flow relatively easier. This indicates body flexibility still induces reduction of shape drag in non-Newtonian fluid flow. Note that the positions and shapes of some plates are close to each other so that they look as if they were overlapped.

Comparison of the four panels on the bottom of Fig. 5 shows that as $n$ increases, the collection of plates with different values of $\hat{K}_{b}$ generates a much smaller overall envelope (representing a much smaller obstacle in the flow). This is related to the fact that the drag increases with the value of $n$. While the smaller envelope in both cases (Fig. 4 and Fig. 5) is caused by the increasing value of $n$, some significant differences are noticed. First, in Fig. 4, each envelop has a "tongue-like" structure at the center which is caused by stretching of the plate at very small generalized Reynolds number $(R e g \leq 0.1)$. The absence of such structure in Fig. 5 is because of the fixed $R e g$ value $(R e g=10)$ for each envelope (formed by different values of plate bending modulus). The other noticeable difference is that the overall envelope size is significantly different in Fig. 5 while they differ only slightly in Fig. 4.

As shown in Figs 3 and 4, the exponent of the power-law of the non-Newtonian fluids has significant influence on the drag coefficient: $C_{d}$ increases with the value of $n$. However, Fig 5 shows that the value of $n$ does not have much effect on the exponent of the power function for drag scaling, particularly for smaller generalized Reynolds numbers $(R e<10)$ : the exponent only slightly increases as the value of $n$ increases.

\section{Summary and Discussion}

We have extended a lattice-Boltzmann (D3Q19 model) based immersed boundary (LBIB) method to the case of non-Newtonian fluids whose constitutive law is described by a power function. The new method is used for simulation of a non-Newtonian fluid flow around a deformable plate supported along its middle line (unconstrained elsewhere) in a threedimensional tunnel. The numerical results suggest that, in the ranges of the dimensionless parameters used in the simulations, 1) the drag coefficient increases with the exponent of the power-law and decreases with the generalized Reynolds number; 2) viscoelastic force dominates the plate drag; 3) the drag scales approximately as 1.9, 1.6, and 1.2 powers of the inflow speed for generalized Reynolds number equal to 1, 10, and 100, respectively. This contrasts sharply with the existing results in literature regarding drag scaling of a body in Newtonian fluid.

The above numerical results of the flexible-plate non-Newtonian-fluid interactions are obtained via analysis from simulations using dimensionless parameters lying on the following ranges: $0.01 \leq R e g \leq 200,0.0001 \leq \hat{K}_{b} \leq 0.008,0.5 \leq n \leq 1.9$. We would like to point out that when these parameters are out of these ranges, i.e., Reg or $\hat{K}_{b}$ is too large, or $n$ is too small, the numerical computation becomes unstable. When $\hat{K}_{b}$ is too large, small plate deformations generate large Lagrangian forces on the fluid; numerical instability may be triggered by the overshoot of the immersed plate due to the large interacting forces. When Reg is too large, the damping effect of viscous forces is significantly lessened; the dominating large inertia forces may causes fast relaxation of the plate to undergo tangential equilibrium. This can induce numerical instability. Moreover, as Reg increases, thinner boundary layers are formed and confined to the plate. Therefore smaller spatial grid spacing and temporal step size are desirable for resolving the flow sufficiently accurately. When $n$ is too small (e.g., 
$n<0.3$ ), the fluid viscosity may becomes too small where the shear rate is large, leading to too big local generalized Reynolds number. Possible synergistic nonlinear effects of these three factors render the numerical analysis of stability more difficulty and intractable. Future efforts on rigorous stability analysis are definitely desired.

Our previous computational study [53] has shown that the drag of a deformable sheet scales approximately as the inflow speed for Newtonian fluid in three dimensions when Reynolds number in the range $[1,100]$. In this study, our results indicate that the drag of the plate scales approximately as 1.9, 1.6, and 1.2 powers of the inflow speed for nonNewtonian fluid when the generalized Reynolds number equals 1, 10, and 100, respectively. The significant difference in the drag scaling may be explained by the non-Newtonian effect such as the fluid elasticity which lacks in a viscous Newtonian fluid. Viscoelastic fluid may induce more drag. Such non-Newtonian effect is incorporated in the generalized Reynolds number Reg; but it lacks in the definition of Reynolds number $(R e)$ for Newtonian fluid.

While drag data of a rigid body (such as a sphere or other particles) in a non-Newtonian fluid are available in literature (see a recent review paper [21]), those of a deformable body in a non-Newtonian fluid are not available, to the best of our knowledge. Nevertheless, our results of drag coefficients versus the generalized Reynolds number (i.e. Fig. 4) are in qualitative agreement with the data of bubbles in a Newtonian fluid and rigid particles in a non-Newtonian. For $R e g \in[0.1,100]$, the range of our drag coefficient $\hat{C}_{d}$ and the trend of $\hat{C}_{d}$ versus $R e g$ both qualitatively agree with the existing data summarized in [21] (Fig. 3 for bubbles and Fig. 7 for rigid particles ): $\hat{C}_{d}$ monotonically decreases from approximately 100 to 1 as $R e g$ increases from 0.1 to 100 . The qualitative agreement reflects the fact that the drag coefficient is computed in our study when the plate has reached a quasi-steady state where the shape and position are almost static (therefore resembling a rigid body of the same geometry). Note that the body deformability still plays an important role in drag and its scaling. The role is incorporated in the definition of the drag coefficient $\hat{C}_{d}$ (see Section $5.2)$.

Our numerical results show that as Reg increases, the plate equilibrium position expands outwards, i.e., the plate bends less for larger $R e$. This seems to be against our intuition. Intuitively, one may speculate that as $R e$ increases, the inertial forces become greater and dominating, which would cause more bending of the plate and, thus, the plate should shrink inwards and present itself as a smaller obstacle to the flow. However, according to our simulations, this does not happen. Similar Re effect was observed for Newtonian flows as well in both two $[50,51]$ and three [53] dimensions. One may speculate that, as the Re increases, the upperstream flow speed of the plate becomes greater than flow speed right behind the plate, thus the pressure behind the plate becomes greater than pressure in front of the plate. Thus the pressure difference pushes the plate towards the upperstream direction. However, the iso-surfaces of pressure for three typical generalized Reynolds numbers $(\operatorname{Reg}=1,10,100)$ do not confirm the hypothesis. This seems to be an interesting phenomenon which may not permit a simple explanation; it is a nice piece of future work.

From the plate shape shown in Figs 3 and 4, one may see that the viscoelastic force seems to dominate the total drag of the plate. The total drag the plate experiences may be decomposed into shape drag and viscoelastic drag. The former is caused by the shape of the obstacle in the flow; the greater is the effective area (normal to the mainstream), the greater is the resistance or drag. The latter is caused by friction on the surface of the obstacle; 
the more viscoelastic is the fluid, the greater is the surface friction. The effective area in Fig 3 (bottom) gets greater, but the drag coefficient gets smaller as Re increases. Similarly, the effective area in Fig 5 gets smaller, but the drag coefficient gets greater as $n$ increases. Therefore, one may conclude that the viscoelastic force dominates the plate drag in both cases.

The significance of our work may be summarized as follows. On one hand, drag data of a deformable body in a non-Newtonian fluid are not available in literature; our computational results fill part of the gap and hence is important in the theory of non-Newtonian fluid. On the other hand, our results may have practical implications. Two possible examples are given below. Firstly, shear thickening fluid may be used for manufacturing sport protective clothing or liquid body armor. When impacted by an external object such as a bullet, the external stress causes the stiffening of fluid by increasing viscosity; the more drag the hit portion of clothing/armor experiences, the more effective the protection is. Our results on Figs. 3 and 4 show that the drag coefficient increases with the value of $n$ for shear thickening fluid. Therefore, these results suggest that a shear-thickening fluid with higher value of $n$ should be preferred for this purpose. Second example is related to the damage of red blood cells in blood flow through stenosed blood vessels. In badly narrowed blood artery inflicted by atherosclerosis, blood rushes through the stenosis. One may assume that the damage is caused by the significantly elevated flow speed and shear rate when cells pass through stenosis. However, in a severely stenosed blood artery, blood stream may get significantly slowed down in the upper and down streams of the stenosis location. Blood is shear thinning. The reduced shear rate in these regions causes increase in viscosity which leads to decrease in Reynolds number. Our results suggest smaller Reynold numbers induce greater drag. Therefore, presence of stenosis may damage the circulating cells in regions away from the stenosis as well.

Acknowledgements We thank the PhD student MinMiao Wang at University of Science and Technology of China for help with flow visualization addressing three unknown Referees' comments and suggestions. L Zhu thanks the US National Science Foundation (NSF) for the support through the grant DMS-1522554. Y Cheng thanks the National Science Foundation of China (NSFC) for the support under the grant 11172219. 


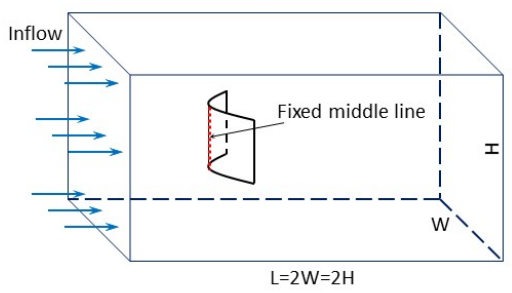

Figure 1: The setup of model problem.

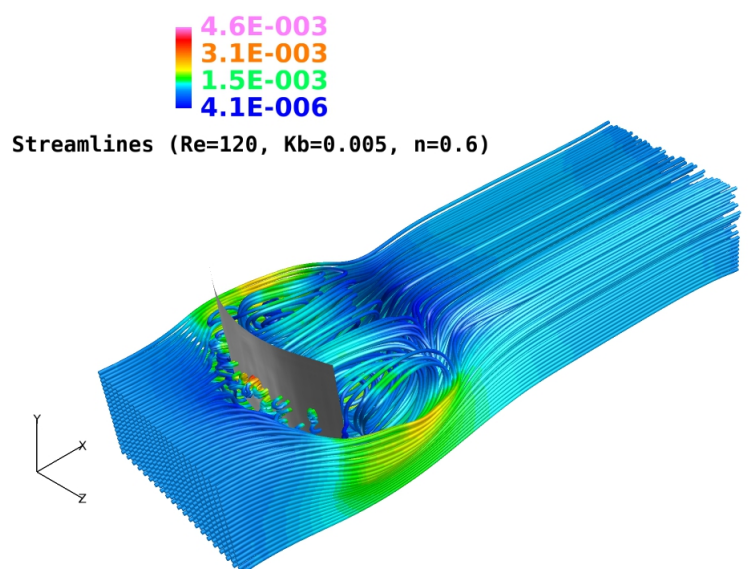

Figure 2: 3D flow visualization through streamlines. 

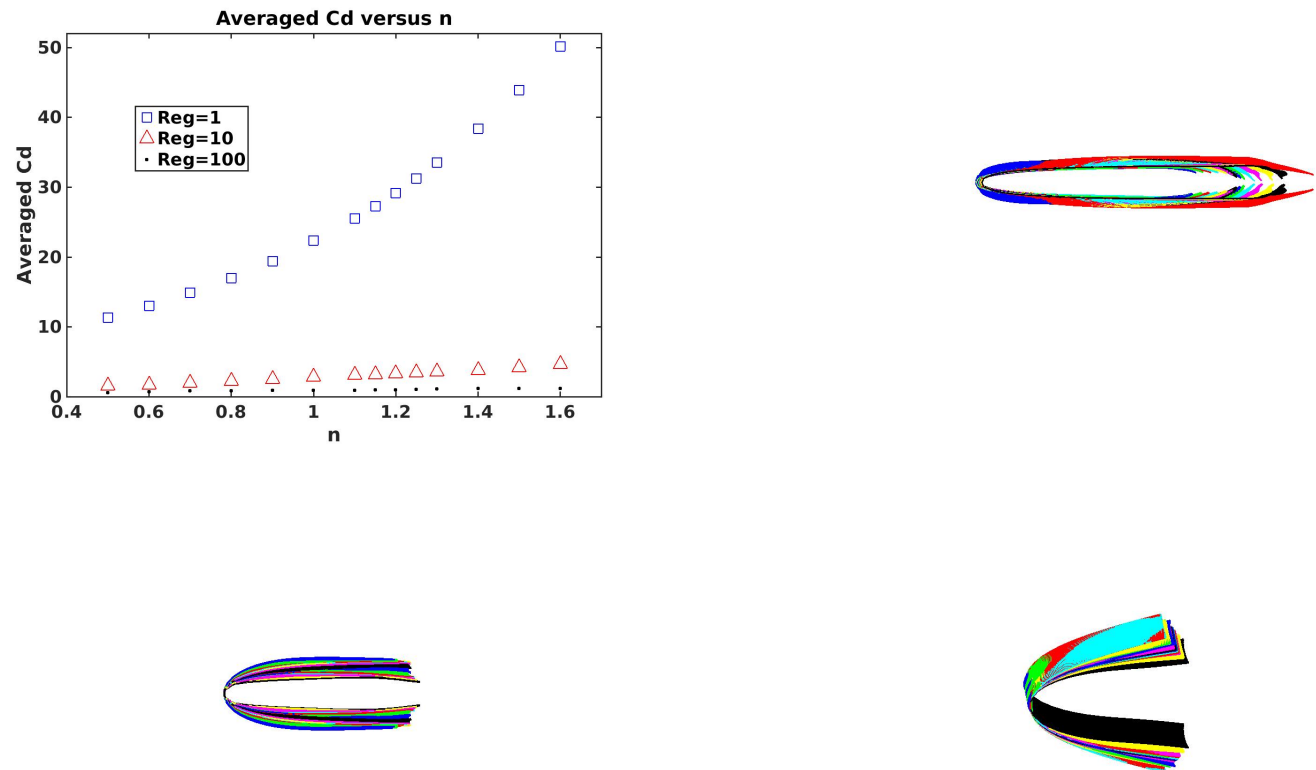

Figure 3: Drag coefficient versus $n$ for three different values of Reynolds number(top) and the position and shape of the plate versus $n$ for $\operatorname{Reg}=1,10,100$ (bottom). Each color represents the position/shape of a plate. Since the available colors are limited in Matlab, a same color may be used twice or more to represent plates of different value $n$. 

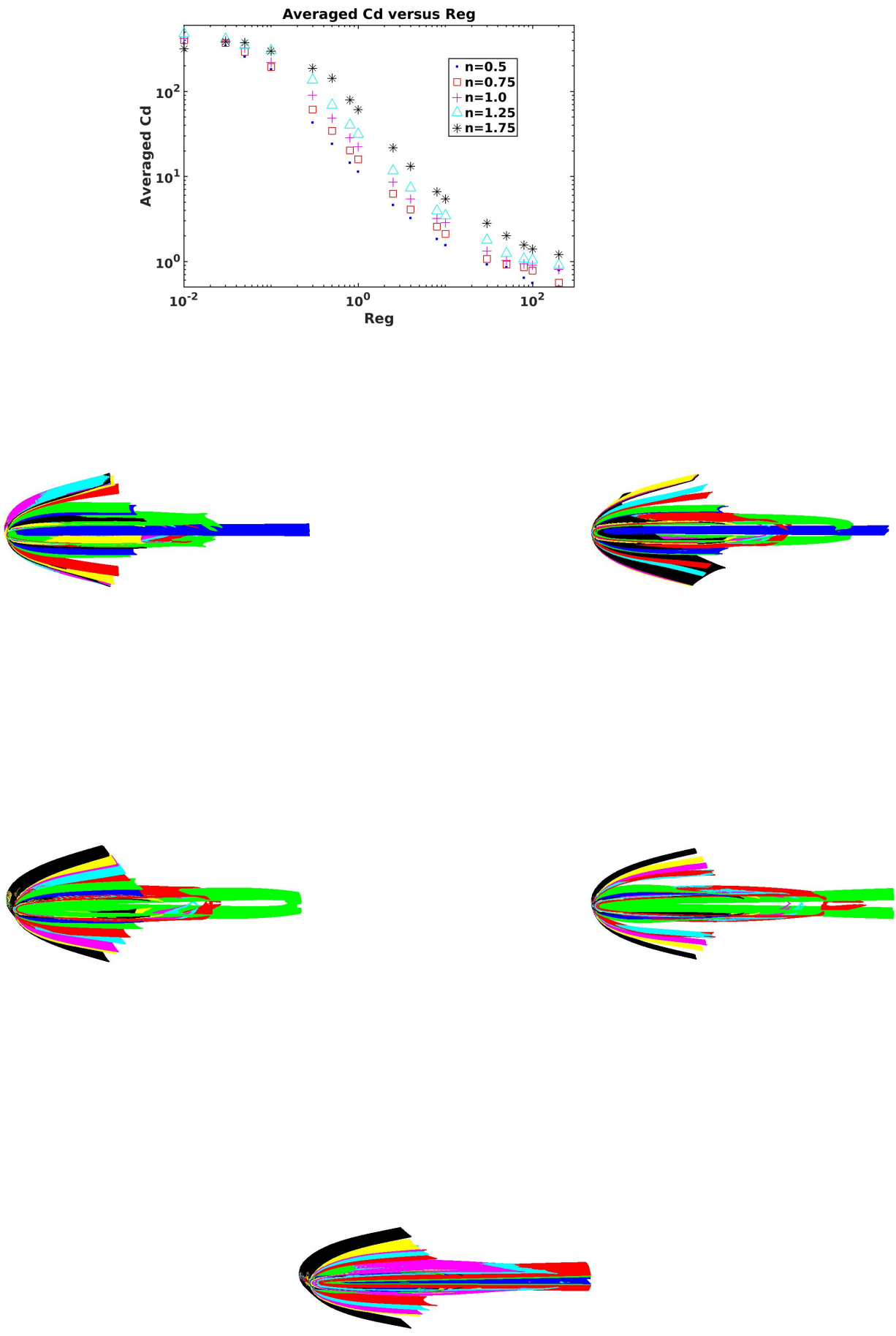

Figure 4: Drag coefficient versus $R e$ for five different values of $\mathrm{n}$ (top) and the shape/position of the plate versus $R e$ for $n=0.5,0.75,1.0,1.25,1.75$ (bottom). Each color represents the position/shape of a plate. Since the available colors are limited in Matlab, a same color may be used twice or more to represent plates of different value Re. 

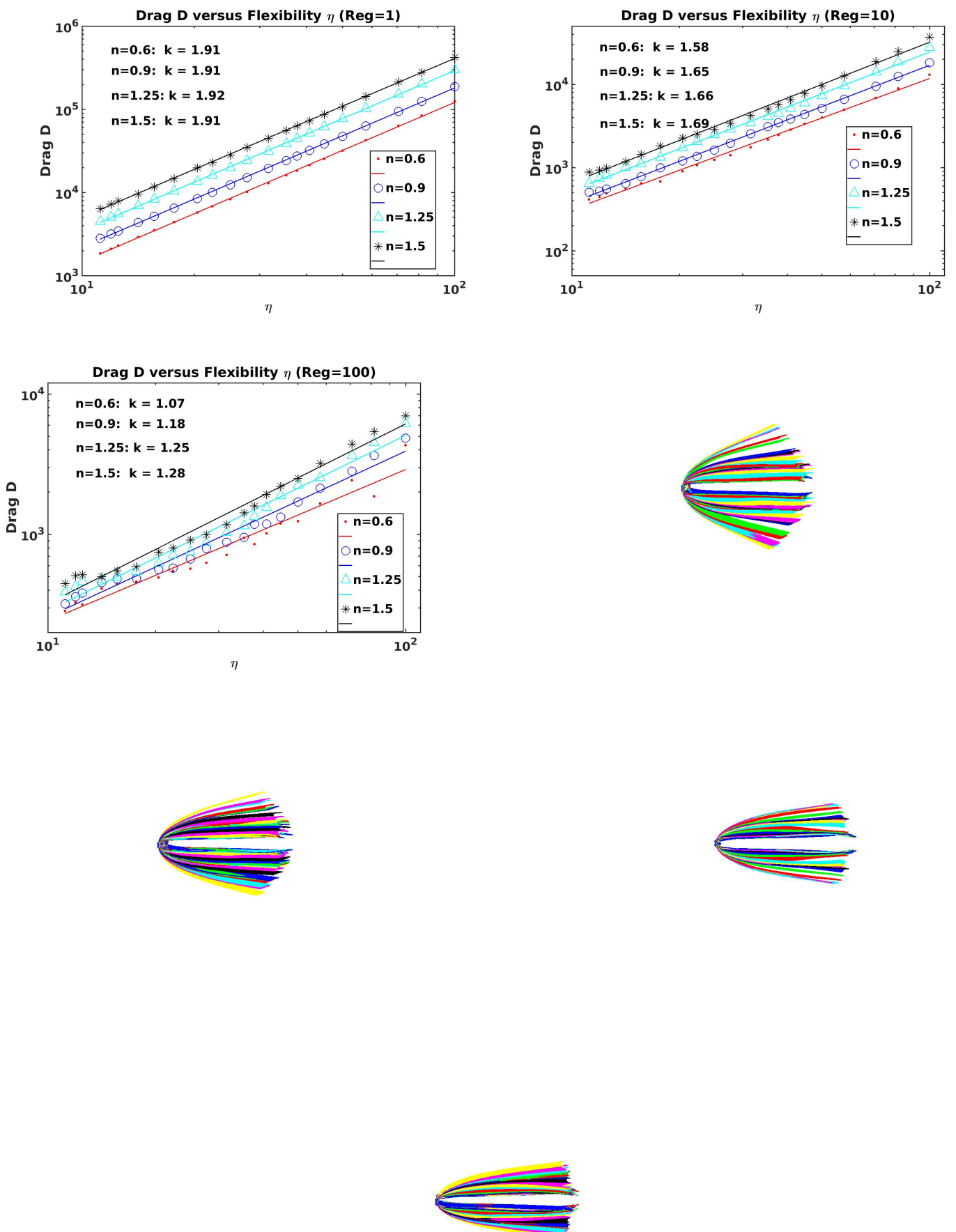

Figure 5: Drag scaling for three values of Reg (top) and the position and shape of the plate versus bending modulus for four different values of $n$ (bottom). The variable $k$ is the exponent of the power-law function between $D$ and $\eta$. The shape/position profiles look similar for other Reynolds numbers . Each color represents the position/shape of a plate. Since the available colors are limited in Matlab, a same color may be used twice or more to represent plates of different value $\hat{K}_{b}$. 


\section{References}

[1] S. Alben, M. Shelley, and J. Zhang, "Drag reduction through self-similar bending of a flexible body", Nature 420, page 6915 (2002).

[2] S. Alben, M. Shelley, and J. Zhang, "How flexibility induces streamlining in a two-dimensional flow ", Phys. of Fluids 16, no. 5, page 1694, May (2002).

[3] P. L. Bhatnagar, E. P. Gross, M. Krook, "A model for collision processes in gases, I: small amplitude process in charged and neutral one-component system", Phys. Rev. , 94, p511, (1954).

[4] I. Borazjani, L. Ge and F. Sotiropoulos, "Curvilinear immersed boundary method for simulating fluid structure interaction with complex 3D rigid bodies", J. Comput. Phys. 227 (16), pp.7587-7620 (2008).

[5] G.K. Batchelor, "An introduction to fluid dynamics", Cambridge Univ. Press, Cambridge (1967).

[6] S. Y. Chen , H. D. Chen, D. Martinez, W. Matthaeus, "Lattice Boltzmann model for simulation of magnetohydrodynamics", Phys. Rev. Lett., 67, p3776, (1991).

[7] S.Y. Chen, D. Martinez, RW Mei, "On boundary conditions in lattice Boltzmann methods", Phys. Fluids, 8 (9): p2527-2536 SEP (1996).

[8] S. Y. Chen, G. D. Doolen, "Lattice Boltzmann Method for fluid flows", Annu Rev. Fluid Mech., 30, p329, (1998).

[9] Y. Cheng and H. Zhang "Immersed boundary method and lattice Boltzmann method coupled FSI simulation of mitral leaflet flow". Computers \& Fluids, accepted, doi:10.1016/j.compfluid.2010.01.003 (2010).

[10] Chrispell, J. C., et al. "Shape oscillations of a droplet in an Oldroyd-B fluid. Physica D Nonlinear Phenomena 240 (20):1593-1601 (2011).

[11] Chrispell, J. C., L. J. Fauci, and M. Shelley. "An actuated elastic sheet interacting with passive and active structures in a viscoelastic fluid. Physics of Fluids 25 (25):441-452 (2013).

[12] Z.G. Feng and E.E. Michaelides, "The immersed boundary-lattice Boltzmann method for solving fluidparticles interaction problems", J. Comput. Phys. 195, 602-628 (2004).

[13] Z.G. Feng and E.E. Michaelides, "Proteus: a direct forcing method in the simulations of particulate flows", J. Comput. Phys. 202, 20-51 (2005).

[14] O. Filippova, S. Succi, F. Mazzocco, C. Arrighetti, G. BElla, and D. Hanel, " Multiscale lattice Boltzmann schemes with turbulence modeling", 170, p. 812 (2001).

[15] B.E. Griffith, C.S. Peskin, "On the order of accuracy of the immersed boundary method: Higher order convergence rates for sufficient smooth problems", J. Comput. Phys. 208, No. 1, pp.75-105, Sept (2005).

[16] Z. Guo, C. Zheng, and B. Shi, "Discrete lattice effects on the forcing term in the lattice Boltzmann method", Phy. Rev. E, 65 (4), 046308 (2002).

[17] X. He, L.-S. Luo, "Theory of lattice Boltzmann method: from the Boltzmann equation to the lattice Boltzmann equation", Phys. Rev. E, 56, p6811, (1997).

[18] X. He and L.-S. Luo, "A priori derivation of the lattice Boltzmann equation", Phys. Rev. E, 55, no. 6, June (1997).

[19] X. He, S. Chen, R. Zhang, "A lattice Boltzmann scheme for incompressible multiphase flow and its application in simulation of Rayleigh-Taylor instability", J. Comput. Phys., 152, p642-663 (1999). 
[20] S. Hou, "Lattice Boltzmann method for incompressible viscous flow", PhD thesis. Kansas State Univ., Manhattan, Kansas.

[21] G.I. Kelbaliyev, "Drag coefficients of variously shaped solid particles, drops, and bubbles." Theoretical Foundations of Chemical Engineering 45.3 (2011): 248-266.

[22] Y. Kim and C.S. Peskin, "Penalty immersed boundary method for an elastic boundary with mass", accepted, Phys. of Fluids, (2007).

[23] J. Kim and P. Moin. "Application of a fractional-step method to incompressible Navier-Stokes equations. ", Journal of computational physics $\mathbf{5 9 . 2}$ (1985): 308-323.

[24] G. Le and J. Zhang, "Boundary slip from the immersed boundary lattice Boltzmann models", Phy. Rev. E 79, 026701 (2009).

[25] M.C. Lai and C.S. Peskin, "An immersed boundary method with formal second order accuracy and reduced numerical viscosity", J. Comput. Phys. 160,705 (2000).

[26] R.J. LeVeque and Z.L. Li, "The immersed interface method for elliptic equations with discontinuous coefficients and singular sources", SIAM J. Numer. Anal. 31, p.1019-1044 (1994).

[27] Z.L. Li and M.C. Lai, "Immersed interface methods for Navier-Stokes equations with singular forces", J. Comput. Phys. 171, p. 822-842 (2001).

[28] Z.L. Li, "The immersed interface method-numerical solutions of PDEs involving interfaces and irregular domains", SIAM press, Philadelphia, (2006).

[29] W.K. Liu, D.K. Kim, and S. Tang, "Mathematical foundations of the immersed finite element method", Comput. Mech., DOI 10.1007/s00466-005-0018-5 (2005).

[30] L.-S. Luo. Unified Theory of the lattice Boltzmann models for nonideal gases. Phys. Rev. Lett. 81: 1618 (1998).

[31] L.-S. Luo, "Theory of the lattice Boltzmann method: Lattice Boltzmann models for nonideal gases", Physical Review E 62(4), pp.4982-4996 (2000).

[32] R. Mittal and G. Iaccarino, "Immersed boundary methods",Annu. Rev. Fluid Mech.37, pp. 239-261, (2005).

[33] Mofrad, Mohammad RK, and Roger D. Kamm, eds. "Cytoskeletal mechanics: models and measurements in cell mechanics". Cambridge University Press (2006).

[34] Y. Peng and L.-S. Luo, "A comparative study of immersed-boundary and interpolated bounce-back methods in LBE", Prog. in Comput. Fluid. Dyn. 8 (1-4), pp.156-167 (2008).

[35] C.S. Peskin, "Flow patterns around heart valves: a numerical method", J. Comput. Phys. 25,220(1977).

[36] C.S. Peskin, "The Immersed Boundary Method", Acta Numerica 11, 479 (2002).

[37] C.J. Pennycuick, "Flying Machines. (Book Reviews: Bird Flight Performance. A Practical Calculation Manual.)." Science 248 (1990).

[38] Y. H. Qian, "Lattice gas and lattice kinetic theory applied to the Navier-Stokes equations", $\mathrm{PhD}$ thesis. University Pierre et Marie Curie, Paris, (1990).

[39] S. Succi, "The lattice Boltzmann equation", Oxford Univ Press, Oxford (2001). 
[40] Y. Sui, Y.T. Chew, P. Roy, and H.T. Low, "A hybrid immersed-boundary and multi-block lattice Boltzmann method for simulating fluid and moving-boundaries interactions", INTERNATIONAL JOURNAL FOR NUMERICAL METHODS IN FLUIDS 53 (11), pp. 1727-1754 (2007)

[41] J. Teran, L. Fauci, and M. Shelley. "Peristaltic pumping and irreversibility of a Stokesian viscoelastic fluid." Physics of Fluids 20.7 (2008): 073101.

[42] F. Tian, "Deformation of a Capsule in a Power-Law Shear Flow." Computational and Mathematical Methods in Medicine 2016, Article ID 7981386, (2016).

[43] H.S. Udaykumar, W. Shyy, M.M Rao, " A mixed Eulerian-Lagrangian method for fluid flows with complex and moving boundaries", Int. J. Numer. Methods Fluids,22, p.691 (1996).

[44] D. Weihs, "Hydromechanics of fish schooling", Nature 241, pp. 290-291 (1973).

[45] D. A. Wolf-Gladrow, "Lattice-gas cellular automata and lattice Boltzmann Models - an introduction", (Springer, Berlin, 2000).

[46] T Ye, R. Mittal, H.S. Udaykumar, W. Shyy, " An accurate Cartesian grid method for viscous incompressible flows with complex immersed boundaries", J. Comput. Phys., 156, p.209 (1999).

[47] H. Yu, S.S. Girimaji, and L.S. Luo, "DNS and LES of decaying isotropic turbulence with and without frame rotation using lattice Boltzmann method", J. Comput. Phys. 209, p.599 (2005).

[48] Y.C. Fung, "Biomechanics: Mechanical properties of living tissues", Springer, New York (1993).

[49] Luoding Zhu, Derek Tretheway, Linda Petzold and Carl Meinhart, "Simulation of fluid slip at hydrophobic microchannel walls by the lattice Boltzmann method", J. Comput. Phys. 202(1), pp. 181-195 (2005).

[50] L. Zhu and C.S. Peskin, "Drag of a flexible fiber in a 2D moving viscous fluid", Computers \& Fluids 36, p.398-406 (2007).

[51] L. Zhu, "Viscous flow past an elastic fibre tethered at its center point: vortex shedding", J. Fluid Mech., 587, pp. 217-234 (2007).

[52] L. Zhu, "Scaling laws for drag of a compliant body moving in an incompressible viscous fluid", J. Fluid Mech. 607, pp. 387-400 (2008).

[53] Luoding Zhu, Guowei He, Shizhao Wang, Laura Miller, Xing Zhang, Qian You and Shiaofeng Fang, "An immersed boundary method by the lattice Boltzmann approach in three dimensions with application", Computers and Mathematics with Applications 61, p.3506-3518 (2011).

[54] L. Zhang, A. Gersternberger, X. Wang, and W.K. Liu, "Immersed finite element method", Comput. Methods Appl. Mech. Eng., 193, p.2051 (2004). 